\title{
Acquired diaphragmatic hernia after hepatectomy and liver transplantation in adults and children: A case series and literature review
}

\author{
Valentine Martin', Emilie Gregoire ${ }^{2}$, Sophie Chopinet ${ }^{2}$, Olivier Scatton ${ }^{3}$, \\ Rémi Dubois ${ }^{4}$, Antoinette Lasseur ${ }^{1}$, Jérôme Dumortier ${ }^{1,5}$, Olivier Boillot ${ }^{1,2,5}$ \\ 'Department of Digestive Diseases, Edouard Herriot Hospital, Hospices Civils de Lyon, Lyon, France, \\ ${ }^{2}$ Department of General Surgery and Liver Transplantation, Aix-Marseille University, \\ Assistance Publique des Hôpitaux de Marseille, la Timone Hospital, Marseille, France, \\ ${ }^{3}$ Department of Hepato-Biliary and Pancreatic and Liver Transplantation, AP-HP Pitié-Salpetrière Hospital, Paris, France, \\ ${ }^{4}$ Department of Pediatric Surgery, Femme Mère Enfant Hospital, Hospices Civils de Lyon, Lyon, France, \\ ${ }^{5}$ Department of Medicine, University Claude Bernard Lyon 1, Lyon, France
}

Backgrounds/Aims: Acquired diaphragmatic hernia is a rare complication following liver surgery in adult and pediatric patients. This study aims to describe main features occurring in adult and pediatric patients after liver surgery and report an up-date review of the literature.

Methods: All adult and pediatric patients who were diagnosed with postoperative acquired diaphragmatic hernia in Lyon and Marseille University Hospitals were included in this study. Diagnosis, clinical, radiologic, and therapeutic data were analysed retrospectively from medical papers and/or electronic records.

Results: Thirteen adults with a median age of 50 years (range, 30-67 years) and 5 children aged 2.4 years (range, 0.9-4 years) were diagnosed with acquired diaphragmatic hernia after a median time of 65.1 (range, 1.8-244.7) and 2 (range, 0.33-10.9) months, respectively, following surgeries (5 live-donor right hepatectomies, 5 right and 1 left hepatectomies for tumors and cysts, and 2 whole liver transplantations in adults; and 5 liver transplantations with left lateral section in children). Eleven patients presented digestive and/or thoracic symptoms whereas seven were asymptomatic and diagnosed by routine imaging follow-up. All patients were re-operated with a median delay of 2.4 months (range, 0-25.3 months) for adults and 1 day (range, 0-2 days) for children. Two recurrences resulted in a secondary surgical repair.

Conclusions: Acquired diaphragmatic hernia is a rare and potentially serious event after liver surgery. Recognition and surgical repair of this particular complication should be considered in the setting of unexplained abdominal and/or thoracic symptoms. Preventive measures should be taken intraoperatively.

Key Words: Hernia, diaphragmatic; Hepatectomy; Liver transplantation; Adults and children; Surgery

Received: March 14, 2021, Revised: April 22, 2021,

Accepted: May 3, 2021

\section{Corresponding author: Olivier Boillot}

Department of Digestive Diseases, Edouard Herriot Hospital, Hospices Civils de Lyon, Pavillon D, 69437 Lyon Cedex 03, France

Tel: +33-4-72-11-62-91, Fax: +33-4-72 -11-67-83,

E-mail: olivier.boillot@chu-lyon.fr

ORCID: https://orcid.org/0000-0002-5819-6312

Copyright (C) The Korean Association of Hepato-Biliary-Pancreatic Surgery This is an Open Access article distributed under the terms of the Creative Commons Attribution Non-Commercial License (http://creativecommons.org/licenses/by-nc/4.0) which non-commercial use, distribution, and reproduction in any medium, provided the original permits unrestricted work is properly cited.

\section{INTRODUCTION}

Acquired diaphragmatic hernia (ADH) is a rare complication following liver surgery in adult and pediatric patients. In the literature, a few low case series have been reported. Its incidence is estimated to be between $0.74 \%$ and $2.9 \%$ [1-12]. Main descriptions of $\mathrm{ADH}$ concern liver resection for tumours, living donor hepatic donation in adult patients, and partial liver graft transplantation in children. Most ADHs have occurred in the right diaphragm after right hepatectomy (RH) in adults and transplantation of a left lateral section (LLS) in pediatric 
recipients in whom the right diaphragmatic space is left uncovered by the liver. Mechanisms ADH are still a matter of debate. Various contributing factors have been proposed, including cautery-related thermal injury, fragile diaphragmatic tissue, poor nutritional status, and factors that can increase intra-abdominal pressure such as postoperative ileus, ascites, pregnancy, obesity, over-size liver graft, and chronic cough. The more frequent organs involved in $\mathrm{ADH}$ are small bowel and colon. Consequently, digestive and/or thoracic symptoms are common, including pain, bowel obstruction or perforation, sepsis, and respiratory distress occurring with various delays after an initial liver surgery. However, a significant proportion of patients are asymptomatic, rendering its diagnosis based only on systematic controlled screenings. ADH probably requires

Table 1. Characteristics of patients undergoing surgical treatment for ADH

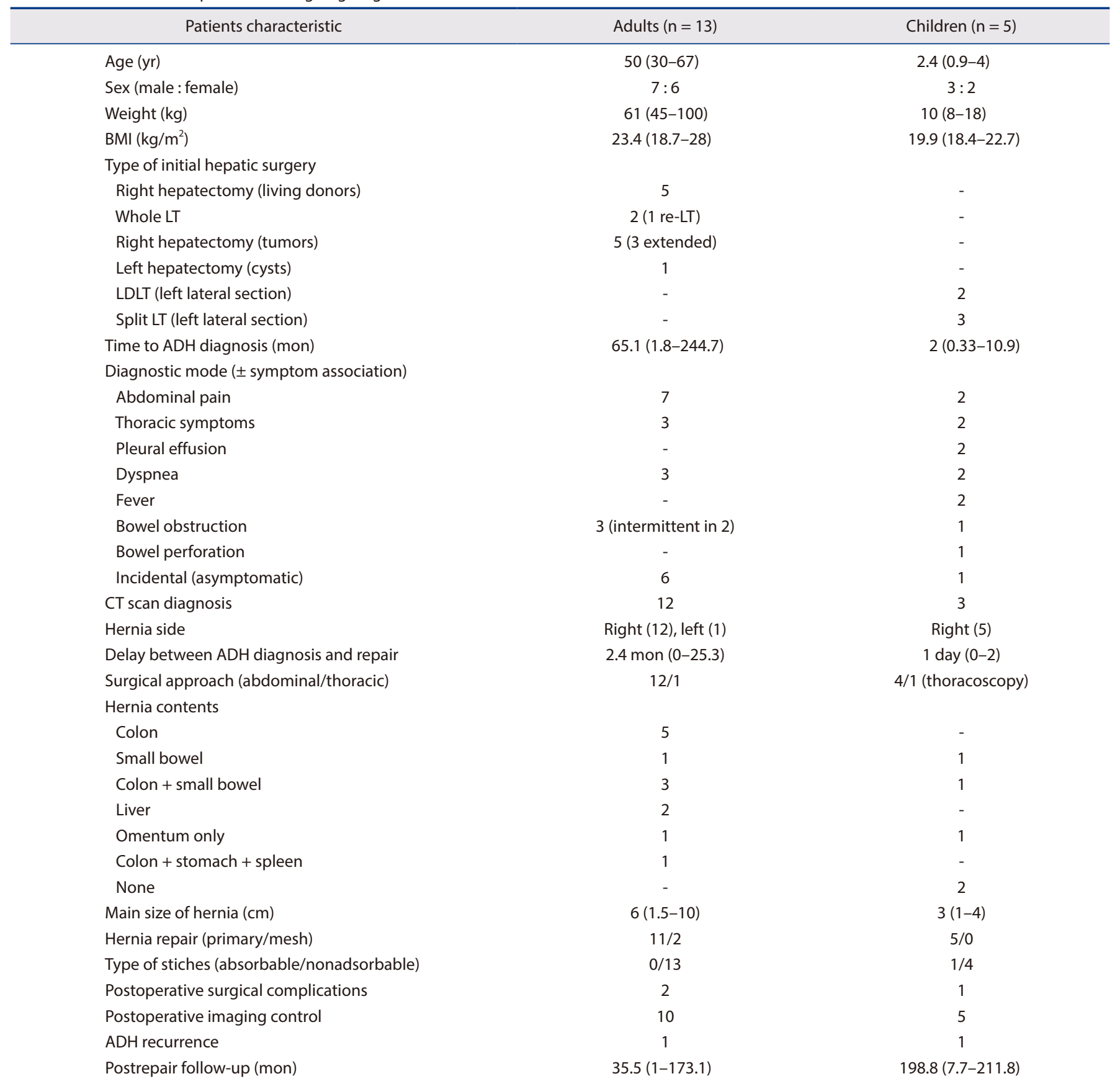

Values are presented as median (range) or number only.

$\mathrm{ADH}$, acquired diaphragmatic hernia; BMI, body mass index; LT, liver transplantation; LDLT, living donor liver transplantation; CT, computed tomography. 
more or less urgent surgical repair according to the intensity of symptoms. Nevertheless, its decision making, surgical approaches, and techniques of repair procedure are not well standardized yet. The aim of the present study was to describe main features of ADH occurring in 18 adult and pediatric patients over a 17-year period with an up-to-date review of available literature.

\section{PATIENTS AND METHODS}

All adult and pediatric patients who presented with $\mathrm{ADH}$ after a liver surgery in Lyon and Marseille University Hospitals were retrospectively included in this study. Informed consent was obtained from each patient or parent before surgery. Data were retrospectively collected from medical papers and/or electronic records. Demographic characteristics and clinical data were reviewed. Diagnosis of ADH was made upon suggestive abdominal and/or thoracic clinical symptoms and imaging, mainly chest X-ray and computed tomography (CT) scan. In some cases, asymptomatic ADH was discovered at the time of protocol follow-up or reoperation for another indication. Decision making and timing (urgent, delayed) for surgical treatment of $\mathrm{ADH}$ were based on the intensity of clinical symptoms (high, low chronic, none) and the amount of incarcerated organs. The surgical abdominal approach was our first choice because it allowed precise checking of the abdominal cavity. Nonetheless, a thoracic (or thoracoscopic) approach seemed appropriate in some cases with herniated part of the liver or small amounts of digestive organs. Primary hernia repair using nonabsorbable interrupted sutures was favoured even in large diaphragmatic defects $(<10 \mathrm{~cm})$. Postoperative controls with clinical evaluation and chest X-rays or CT were made at three months. Thereafter, they were performed as part of usual follow-up of patients for their initial disease and living donor surveillance. A literature search was performed through MEDLINE (PubMed) using the following keywords: "diaphragmatic hernia" or "diaphragmatic defect" or "acquired diaphragmatic hernia."

\section{Statistical analysis}

Quantitative variables were described using median and interquartile range (IQR). Qualitative values were tabulated and percentages were calculated. All statistical analyses were performed with IBM SPSS version 23.0 (IBM Corp., Armonk, NY, USA).

\section{RESULTS}

\section{Study population}

Between January 2003 and October 2020, 18 patients who had previous hepatic surgeries in Lyon (14 cases) and Marseille (4 cases) University hospitals were diagnosed with $\mathrm{ADH}$. There were 13 adults with a median age of 50 years (range,
30-67 years) and 5 children aged 2.4 years (range, $0.9-4$ years). Median weight and body mass index at the time of $\mathrm{ADH}$ diagnosis were $61 \mathrm{~kg}$ (range, $45-100 \mathrm{~kg}$ ) and $23.4 \mathrm{~kg} / \mathrm{m}^{2}$ (range, $18.7-28 \mathrm{~kg} / \mathrm{m}^{2}$ ) for adult patients and $10 \mathrm{~kg}$ (range, $8-18 \mathrm{~kg}$ ) and $19.9 \mathrm{~kg} / \mathrm{m}^{2}$ (range, $18.4-22.7 \mathrm{~kg} / \mathrm{m}^{2}$ ) for pediatric patients, respectively. In adults, previous surgery included five open $\mathrm{RH}$ for live donation, 2 whole liver transplantations (LT), $5 \mathrm{RH}$ (3 extended) for hepatocellular carcinoma (HCC) (2 cases), giant haemangioma (1 case), hepatic adenomatosis (1 case), epithelioid haemangioendothelioma (1 case), and 1 left hepatectomy (LH) for polycystic disease. Split LT and living donor LT with LLS were performed in 3 and 2 children, respectively, for biliary atresia (2 cases), progressive familial intrahepatic cholestasis, or Alagille and Budd Chiari syndromes. None of these patients was obese. In transplanted children, the median graft-to-recipient weight ratio (GRWR) was 3.1\% (IQR: 1.5\%$3.6 \%$ ). Clinical characteristics of patients are summarized in Table 1.

\section{Diagnosis}

The median time between hepatic surgery and diagnosis of ADH was 65.1 months (range, 1.8-244.7 months) for adult patients and 2 months (range, $0.33-10.9$ months) for pediatric patients. Diagnosis was made by chest X-ray and CT in 11 patients for exploration of digestive and/or thoracic symptoms located on the right side in 10 patients and on the left side in one patient. One child who had digestive liquid exiting through a thoracic drainage after LT immediately underwent surgical revision without previous CT. Symptoms were considered of high intensity in five patients ( 1 adult and 4 children) and of low chronic intensity in dix adult patients.

In seven asymptomatic patients, $\mathrm{ADH}$ was discovered by imaging protocol controls after $\mathrm{RH}$ for living donation (2 cases), $\mathrm{RH}$ ( 2 cases) for tumor, LT (1 case), during retransplantation (1 child), or during reoperation for recurrent HCC inside the liver (1 adult) after a previous RH.

Fig. 1 shows CT-scan and operative views of a right $\mathrm{ADH}$ containing digestive elements in a live donor before and after a diaphragmatic repair. In an asymptomatic transplanted patient who had a whole liver graft 20 years ago, systematic protocol CT demonstrated part of the liver herniated into the pleural space and its resolution after surgery through a thoracotomy approach (Fig. 2).

Of note, two patients were diabetic. After the initial liver surgery, three patients presented persistent ascites (1 adult and 2 children) and two live donor females had two pregnancies each.

\section{Surgical decision making and timing}

Once the diagnosis of ADH was established, our policy was to systematically perform a surgical repair. According to clinical presentation, patients with high intensity symptoms required reoperation in emergency while those with low 

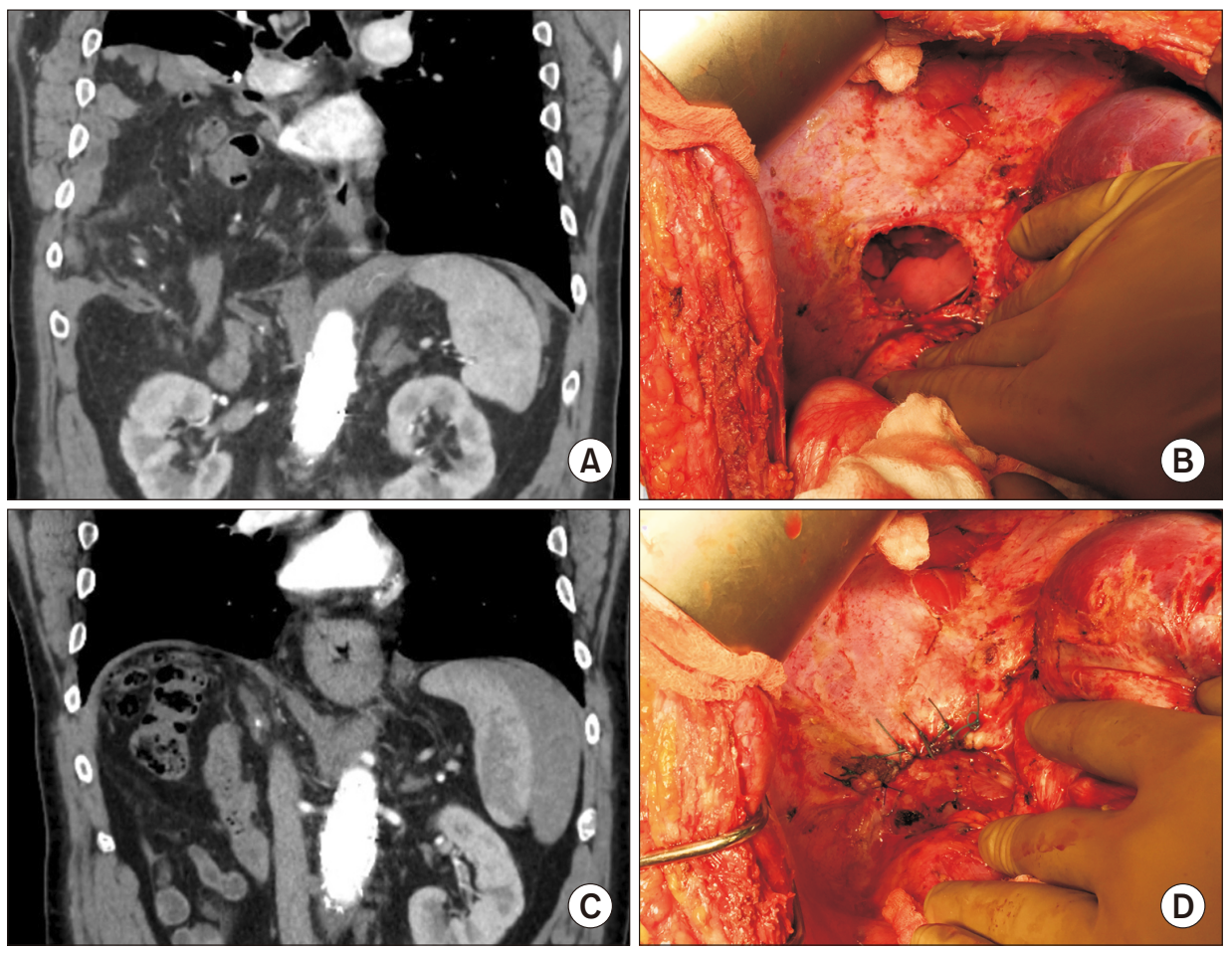

Fig. 1. Computed tomography scan and operative views of a right acquired diaphragmatic hernia containing digestive elements in a live donor before $(A, B)$ and after a diaphragmatic repair (C, D).
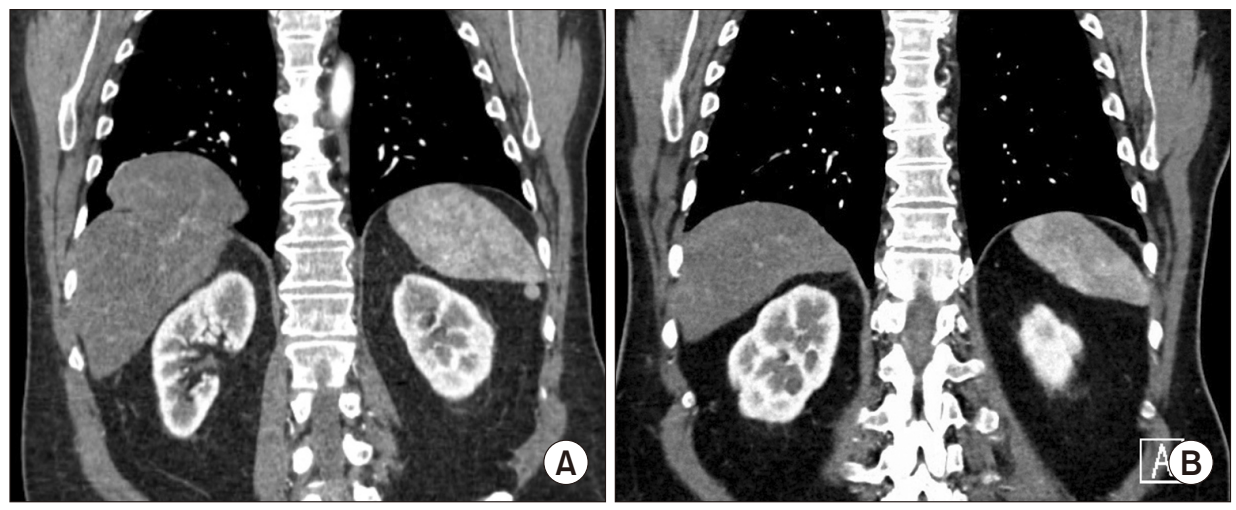

Fig. 2. Systematic protocol computed tomography-scan of an asymptomatic transplanted patient who had a whole liver graft 20 years ago, demonstrating part of the liver herniated into the pleural space (A) and its resolution after surgery through a thoracotomy approach (B).

chronic symptoms underwent delayed surgical repair after their initial surgery in a median delay of 3.8 months (range, 0.6-17.2 months). Asymptomatic patients were re-operated at 2.4 months (range, 0-25.3 months) after their initial surgery. Overall, median delay from $\mathrm{ADH}$ diagnosis to surgical repair was 2.4 months (range, 0-25.3 months) for adult patients and 1 day (range, 0-2 days) for pediatric patients (Table 1).

\section{Surgical procedures and findings}

Sixteen patients were re-operated with an open abdominal approach. Surgeries were performed through thoracotomy in one adult and thoracoscopy in one child. All but one ADH were located at the right posteromedial region at the bare area with more or less extension to the right of the diaphragm whereas it was in the middle part of the left diaphragm on the line of the left triangular ligament attachment in the remaining patients who underwent LH. No patient had diaphragmatic perforation or resection during the initial hepatic procedure. Median sizes of $\mathrm{ADH}$ were $6 \mathrm{~cm}$ (range, $1.5-10 \mathrm{~cm}$ ) and $3 \mathrm{~cm}$ (range, $1-4 \mathrm{~cm}$ ) in adult and pediatric patients, respectively. In adult patients, seven had an ADH size of 5 to $7 \mathrm{~cm}$ and three had an ADH size of 7 to $10 \mathrm{~cm}$. Colon $(\mathrm{n}=5)$, colon and small bowel $(\mathrm{n}=4)$, small bowel $(\mathrm{n}=2)$, liver $(\mathrm{n}=2)$, omentum only $(\mathrm{n}=2)$, and colon + stomach + spleen $(n=1)$ were incarcerated in the thoracic space. In two pediatric cases, hernia did not contain any organ. No patient underwent digestive resection. However, for one pediatric case with colon perforation, a colostomy was performed. After reintegration of digestive organs in the 
abdominal cavity, edges of diaphragmatic defects were slightly recut and sutured with interrupted nonabsorbable sutures (n $=17$, including two adults who had additional mesh repair) or absorbable $(n=1)$ sutures in one child. In all cases but three, an abdominal drainage was left and a thoracic drainage was installed in four patients. The median duration of surgery was 120 minutes (range, 75-420 minutes). The median hospital stay was 8 days (range, 5-228 days). One live donor patient experienced concomitant biliary injury requiring further treatments by endoscopic stenting. One adult patient was re-operated for abdominal bleeding. One child was re-operated for digestive perforation in the context of LT.

\section{Follow-up and outcomes}

Overall median postoperative follow-up was 56.8 months (range, 1-211.8 months). All patients but three were subjected to postoperative imaging controls. All patients remained asymptomatic except for two patients with $\mathrm{ADH}$ recurrence diagnosed after 4.5 and 78.5 months that required urgent reoperations for bowel obstruction.

\section{Incidences of ADH}

Regarding our experience in Lyon (data not available in Marseille), among 130 pediatric LT performed using LLS, the incidence of $\mathrm{ADH}$ was $3.07 \%$. In adult transplant recipients who had received either a whole or a partial liver, $2(0.22 \%)$ out of 902 developed $\mathrm{ADH}$. In living donors who underwent $\mathrm{RH}, 5$ (9.6\%) out of 52 were diagnosed with $\mathrm{ADH}$. This incidence was much lower in 757 patients who had RH (or right extended) or LH (0.66\%).

\section{DISCUSSION}

Our series of adult and pediatric populations with ADH represents the largest number of patients reported by now. By contrast, all other studies have focused on either adult or pediatric patients for a single situation (RH in living donors or tumors or LT in children using LLS or left liver).

Diaphragm is a musculo-aponeurotic septum tensed between the thorax and the abdomen. Its structure is made of smooth muscular fibers converging to a sinew center presenting an orifice for the inferior vena cava. The non-muscular bare area of the right diaphragm located postero-medially is thin $(0.3 \mathrm{~cm}$ versus $1 \mathrm{~cm}$ thick for the muscular part in adults). Therefore, it has fragility with a risk of ADH. The bare area might be more sensible to aggressive maneuvers such as thermic injury caused by electric cautering used during right liver mobilization, potentially leading to devascularization and delayed healing or even perforation. The combination with others factors such as increased abdominal pressure due to post-operative ileus or ascites or pregnancy, oversized left partial liver graft in children, permanent motion of the diaphragm which remains uncovered by the protection of the liver, and poor nutritional status (in children undergoing LT) may contribute to the occurrence of $\mathrm{ADH}$ as multifactorial risk factors. $\mathrm{ADH}$ is a serious event. It may lead to life-threatening complications with intestinal obstruction, perforation, and overwhelming sepsis. In accordance with all authors but $\mathrm{Oh}$ et al. [7], we believe that surgical repair of $\mathrm{ADH}$ is mandatory even for asymptomatic cases with an aim to treat or prevent potential severe complications.

There were differences between our patient populations who developed ADH. Such differences might be due to the following reasons. Our five living donor patients with a median age of 37 years (range, 20-43 years) with normal preoperative nutritional and health statuses did not have any known risk factors. All surgical procedures and immediate postoperative periods were uneventful. Nonetheless, there were ADHs diagnosed after a long median delay of 122.9 months (range, 19.9-205.7 months). Three were symptomatic, including two donor females after two pregnancies each. It should be noted that the incidence of $\mathrm{ADH}$ in our series was much higher (9.6\%) than that reported in the literature. A possible explanation was that the excessive use of thermal electric cautery and argon beamer applied to the thin and vulnerable bare area during right liver mobilization in order to secure hemostasis might have led to the development of a secondary $\mathrm{ADH}$, in addition to concomitant increase of post-operative abdominal pressure on an uncovered diaphragm (especially in the two cases of pregnancies). Up to now, 12 publications have described the occurrence of $\mathrm{DH}$ in 22 live donors [1,2,5,7,13-20], with an incidence ranging from $1.6 \%$ to $2.7 \%$ (Table 2). Findings are concordant, as it is often a late complication after $\mathrm{RH}$ in living donor patients caused by chronic elevation of abdominal pressure on an uncovered diaphragm which is subjected to a thermic injury during surgery. Interestingly, in a series of nine live donors who have developed $\mathrm{ADH}$ in a median delay of 5.7 months (range, 3-16.6 months) after RH, Oh et al. [7] did not operate on six asymptomatic live donors for their diaphragmatic defects. Thus, they were observed with repeated $\mathrm{CT}$, showing a progressive increase in size of ADH over time [7]. These observations underline the delayed occurrence and progressive enlargement of defect in a heathy population who undergo $\mathrm{RH}$ for live donation and interrogates about the need for systematic surgical repair. Literature is rather scare about $\mathrm{ADH}$ after $\mathrm{RH}$ for donation probably because of unrecognized cases and the lack of long term follow-up of these patients [13]. Based on observations of Oh et al. [7], surgical repair of asymptomatic ADH should probably be mandatory before defects get larger and symptomatic.

Among our six patients with a median age of 48 years (range, 44-64 years) and normal liver function who underwent major hepatectomies for tumors or cysts, $\mathrm{ADH}$ were discovered at 7.8 months (range, 1.86-30.6 months) after surgery. All presented huge tumors or liver (polycystic) at the initial surgery responsible for difficult and hemorrhagic hepatic mobilization and somehow aggravating possible thermal injury by excessive electrocautery hemostasis to the diaphragm in spite of the use 


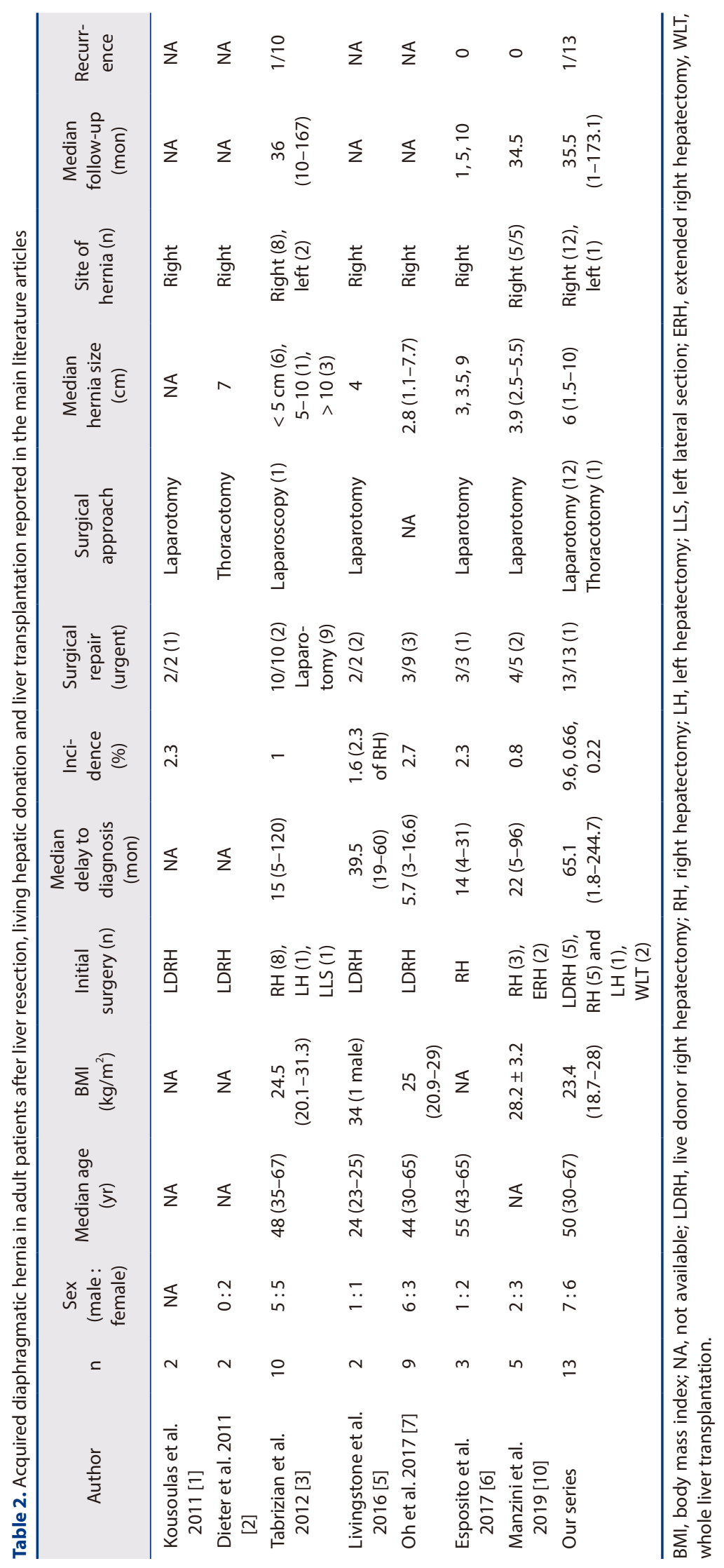




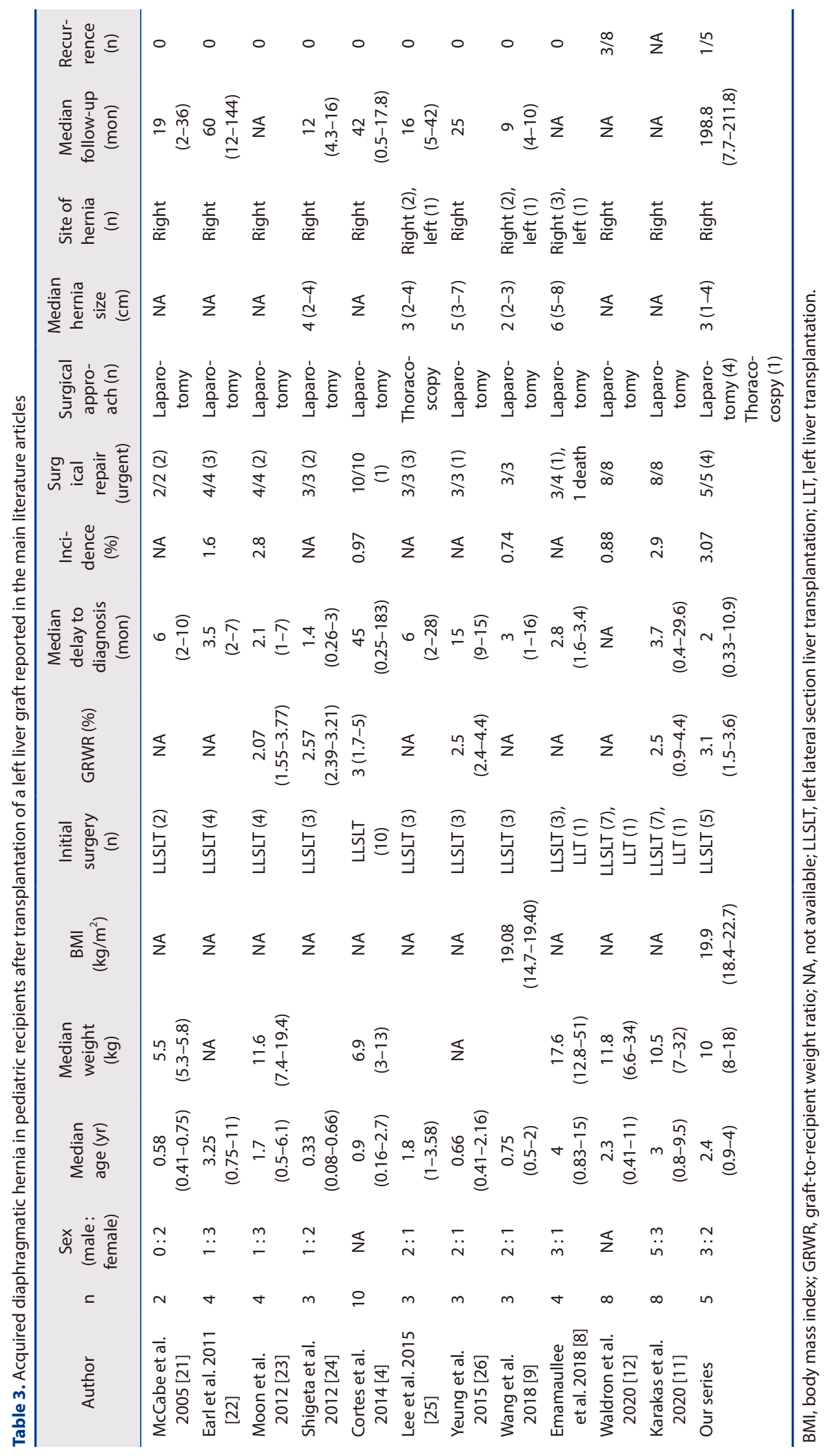


of an anterior approach and banding which is supposed to improve the access to the diaphragm. Moreover, two patients were diabetic known to be a risk factor that could interfere with wound healing. For a 52-year-old woman who underwent a LH for huge polycystic liver disease, mobilization of the left liver was technically difficult because of its rigidity and the lack of visibility which was probably responsible for the enhanced thermal diaphragmatic injury and secondary perforation. These observations weare in accordance with other series $[3,6,10]$, underlining that almost all ADHs occurred after $\mathrm{RH}$ or extended RH in patients with huge tumors that enhanced technical difficulties (Table 2). Nevertheless, the incidence of $\mathrm{ADH}$ remained low at $0.8 \%$ to $2.3 \%[3,6,10]$. It was $0.66 \%$ in our series. In two other adult patients who underwent whole LT, $\mathrm{ADH}$ leading to thoracic liver herniation occurred in spite of the fact that the right diaphragm was covered by the liver. For these cases, we probably can incriminate intraoperative diaphragm injury and postoperative increase abdominal pressure.

In children who developed $\mathrm{DH}$, most of them were small, young, and cirrhotic with impaired coagulation profile and nutritional status when they underwent LT. Table 3 summarizes most relevant publications on 56 children who developed ADH after LT $[4,8,9,11,12,21-26]$. Their median age and weight were 0.75 to 4 years and 5.5 to $17.6 \mathrm{~kg}$, respectively. All children received LLS but left liver in 3 patients. Their median GRWR was within the usual range (2.07\% to $3 \%)$. When compared to adult patients, $\mathrm{ADH}$ seemed to occur more frequently in children and shorter after liver surgery, mainly in the first six months in the majority of cases (Table 3). Moreover, they were more symptomatic with high intensity clinical manifestations. Emergency reoperation was often necessary. Incidences of $\mathrm{ADH}$ were comparable among transplant teams (0.74\% to $2.9 \%)$. Hernias were located in the great majority on the right diaphragm. Their sizes ranged from $2 \mathrm{~cm}$ to $6 \mathrm{~cm}$. Primary surgical repair was achieved in all children with mainly nonabsorbable stiches by laparotomy in most cases compared to thoracoscopy. Most defects were located postero-medially at the site of the bare area. Its thinness and high sensitivity to high use of diathermy were underlined by Karakas et al. [11] and Moon et al. [23]. Many possible risk factors predisposing for $\mathrm{ADH}$ in children after LT have been reported, including the use of left side grafts leaving the right diaphragm uncovered, especially in small infants with coagulopathy and malnutrition [24], over-sized grafts with high GRWR generating increased abdominal pressure which can be aggravated by edema of the intestine caused by vascular clampings and portal hypertension [4,11], difficult and hemorrhagic dissection of adhesions between the liver and the diaphragm after a previous abdominal surgery (i.e., Kasai procedure), vascularization of the fragile and thin bare area leading to partial necrosis and potential perforation, and immunosuppression especially by the use of corticosteroids that could delay healing [11]. The combination of many of these factors probably can enhance the risk of $\mathrm{ADH}$ development.
Nevertheless, in a study of case-matched control group without $\mathrm{ADH}$, Karakas et al. [11] were unable to determine any risk factor responsible for the occurrence of $\mathrm{ADH}$. Up to now, 77 cases (including ours) of ADH after pediatric LT have been identified in 21 articles from the literature $[4,8,9,11,12,21-36]$.

In conclusion, $\mathrm{ADH}$ after liver surgery is a rare, often delayed, and potentially serious event. Recognition of this particular complication should be considered in the setting of unexplained abdominal and/or thoracic symptoms. $\mathrm{ADH}$ seems to occur more frequently with more high intensity symptoms and earlier onset after a primary surgery in children compared to that in adult patients. Intraoperative prevention of this complication should be taken into consideration by careful dissection of the diaphragm with cautious use of thermal devices. Its integrity should be checked before abdominal closure. Surgical repair should be considered after diagnosis of all ADHs, even in asymptomatic cases which can worsen over time.

\section{CONFLICT OF INTEREST}

No potential conflict of interest relevant to this article was reported.

\section{ORCID}

Valentine Martin, https://orcid.org/0000-0002-5721-4873 Emilie Gregoire, https://orcid.org/0000-0001-6576-4405 Sophie Chopinet, https://orcid.org/0000-0002-5674-7280 Olivier Scatton, https://orcid.org/0000-0001-8313-3500 Rémi Dubois, https://orcid.org/0000-0003-1224-5913 Antoinette Lasseur, https://orcid.org/0000-0001-6311-1224 Jérôme Dumortier, https://orcid.org/0000-0002-7824-5396 Olivier Boillot, https://orcid.org/0000-0002-5819-6312

\section{AUTHOR CONTRIBUTIONS}

Conceptualization: VM, JD, OB. Data curation: VM, OB. Methodology: JD, OB. Visualization: VM, EG, SC, OS, AL, JD, OB. Writing - original draft: VM, JD, OB. Writing - review \& editing: All authors.

\section{REFERENCES}

1. Kousoulas L, Becker T, Richter N, Emmanouilidis N, Schrem H, Barg-Hock H, et al. Living donor liver transplantation: effect of the type of liver graft donation on donor mortality and morbidity. Transpl Int 2011;24:251-258.

2. Dieter RA Jr, Spitz J, Kuzycz G. Incarcerated diaphragmatic hernia with intrathoracic bowel obstruction after right liver donation. Int Surg 2011;96:239-244.

3. Tabrizian P, Jibara G, Shrager B, Elsabbagh AM, Roayaie S, Schwartz ME. Diaphragmatic hernia after hepatic resection: case series at a single Western institution. J Gastrointest Surg 2012;16:1910-1914. 
4. Cortes M, Tapuria N, Khorsandi SE, Ibars EP, Vilca-Melendez H, Rela $\mathrm{M}$, et al. Diaphragmatic hernia after liver transplantation in children: case series and review of the literature. Liver Transpl 2014;20:14291435.

5. Livingstone SM, Andres A, Shapiro AM, Kneteman NN, Bigam DL. Diaphragmatic hernia after living donor right hepatectomy: proposal for a screening protocol. Transplant Direct 2016;2:e84.

6. Esposito F, Lim C, Salloum C, Osseis M, Lahat E, Compagnon P, et al. Diaphragmatic hernia following liver resection: case series and review of the literature. Ann Hepatobiliary Pancreat Surg 2017;21:114121.

7. Oh JW, Oh SN, Jung SE, Byun JY. Diaphragmatic hernia after living-donor right hepatectomy: an important late donor complication. J Comput Assist Tomogr 2017;41:726-730.

8. Emamaullee JA, Nekrasov V, Gilmour S, Kneteman N, Yanni G, Kohli R, et al. Case series and systematic review of acquired diaphragmatic hernia after liver transplantation. Pediatr Transplant 2018;22:e13296.

9. Wang K, Gao W, Ma N, Meng XC, Zhang W, Sun C, et al. Acquired diaphragmatic hernia in pediatrics after living donor liver transplantation: three cases report and review of literature. Medicine (Baltimore) 2018;97:e0346.

10. Manzini G, Kuemmerli C, Reiner CS, Petrowsky H, Gutschow CA. Enterothorax after hepatic surgery: a single-center experience. World J Surg 2019;43:902-909.

11. Karakas S, Sahin TT, Kutluturk K, Otan E, Baskiran A, Sarici KB, et al. Diaphragmatic hernias after pediatric liver transplantation: experience of a high-volume transplant center. Pediatr Transplant 2020;24:e13684.

12. Waldron LS, Cerisuelo MC, Lo D, Sayed BA, Vilca-Melendez H, Magliocca J, et al. Diaphragmatic Hernia following Pediatric Liver Transplantation: An Underappreciated Complication Prone to Recur. Eur J Pediatr Surg 2020. https://doi.org/10.1055/s-0040-1716882 [in press]

13. Lochan R, Saif R, Ganjoo N, Sakpal M, Panackal C, Raja K, et al. Diaphragmatic herniation following donor hepatectomy for living donor liver transplantation: a serious complication not given due recognition. Ann Hepatobiliary Pancreat Surg 2017;21:232-236.

14. Hawxby AM, Mason DP, Klein AS. Diaphragmatic hernia after right donor and hepatectomy: a rare donor complication of partial hepatectomy for transplantation. Hepatobiliary Pancreat Dis Int 2006;5:459461.

15. Perwaiz A, Mehta N, Mohanka R, Kumaran V, Nundy S, Soin AS. Right-sided diaphragmatic hernia in an adult after living donor liver transplant: a rare cause of post-transplant recurrent abdominal pain. Hernia 2010;14:547-549.

16. Dieter RA Jr. Postoperative right diaphragmatic hernia with enterothorax in live liver donors. Exp Clin Transplant 2011;9:353.

17. Vernadakis S, Paul A, Kykalos S, Fouzas I, Kaiser GM, Sotiropoulos GC. Incarcerated diaphragmatic hernia after right hepatectomy for living donor liver transplantation: case report of an extremely rare late donor complication. Transplant Proc 2012;44:2770-2772.

18. Mizuno S, Tanemura A, Isaji S. Incarcerated left diaphragmatic her- nia following left hepatectomy for living donor liver transplantation. Transpl Int 2014;27:e65-e67.

19. Jeng KS, Huang CC, Lin CK, Lin CC, Wu JM, Chen KH, et al. Early incarcerated diaphragmatic hernia following right donor hepatectomy: a case report. Transplant Proc 2015;47:815-816.

20. Takaichi S, Takahashi T, Funaki S, Tanaka K, Miyazaki Y, Makino T, et al. Laparoscopic repair of an incarcerated diaphragmatic hernia after right hepatectomy for hepatic injury: a case report. Surg Case Rep 2018;4:135.

21. McCabe AJ, Orr JD, Sharif K, De Ville de Goyet J. Right-sided diaphragmatic hernia in infants after liver transplantation. J Pediatr Surg 2005;40:1181-1184.

22. Earl TM, Wellen JR, Anderson CD, Nadler M, Doyle MM, Shenoy SS, et al. Small bowel obstruction after pediatric liver transplantation: the unusual is the usual. J Am Coll Surg 2011;212:62-67.

23. Moon SB, Jung SM, Kwon CH, Kim SJ, Joh JW, Seo JM, et al. Posteromedial diaphragmatic hernia following pediatric liver transplantation. Pediatr Transplant 2012;16:E106-E109.

24. Shigeta T, Sakamoto S, Kanazawa H, Fukuda A, Kakiuchi T, Karaki $\mathrm{C}$, et al. Diaphragmatic hernia in infants following living donor liver transplantation: report of three cases and a review of the literature. Pediatr Transplant 2012;16:496-500.

25. Lee S, Seo JM, Younes AE, Oh CY, Lee SK. Thoracoscopic approach for repair of diaphragmatic hernia occurring after pediatric liver transplant. Medicine (Baltimore) 2015;94:e1376.

26. Yeung F, Chung PH, Wong KK, Tam PK. Iatrogenic diaphragmatic hernia in paediatric patients. Pediatr Surg Int 2015;31:589-592.

27. Lam HD, Mejia J, Soltys KA, Sindhi R, Mazariegos G, Bond G. Right diaphragmatic hernia after liver transplant in pediatrics: a case report and review of the literature. Pediatr Transplant 2013;17:E77-E80.

28. Bonatti H, Muiesan P, Connelly S, Baker A, Mieli-Vergani G, Gibbs $\mathrm{P}$, et al. Hepatic transplantation in children under 3 months of age: a single centre's experience. J Pediatr Surg 1997;32:486-488.

29. Englert C, Helmke K, Richter A, Beckmann M, Rogiers X, Burdelski $M$, et al. Diaphragmatic hernia resulting in enterothorax following pediatric liver transplantation: a rare complication. Transplantation 2006;82:574-576.

30. Okajima H, Hayashida S, Iwasaki H, Suda H, Takeichi T, Ueno M, et al. Bowel obstruction due to diaphragmatic hernia in an elder child after pediatric liver transplantation. Pediatr Transplant 2007;11:324326.

31. Kazimi M, Ibis C, Alper I, Ulas M, Baran M, Arikan C, et al. Right-sided diaphragmatic hernia after orthotopic liver transplantation: report of two cases. Pediatr Transplant 2010;14:e62-e64.

32. Dökümcü Z, Divarcı E, Erdener A, Sözbilen M, Ergün O. Acquired right diaphragmatic hernia following pediatric living donor orthotopic liver transplantation. Pediatr Transplant 2015;19:E149-E151.

33. Kirnap M, Akdur A, Ozcay F, Soy E, Coskun M, Moray G, et al. Diaphragmatic hernia after pediatric liver transplant. Exp Clin Transplant 2015;13:471-474.

34. Yazdani M, Park E, Udayasankar U. Postsurgical diaphragmatic herniation: a rare delayed complication of pediatric intraabdominal surgery. J Pediatr Surg 2016;51:333-335. 
35. Okur MH, Yankol Y, Mecit N, Hoş G, Ertugrul G, Kanmaz T, et al. Diaphragmatic hernia after liver transplant in children: report of 2 cases. Exp Clin Transplant 2018;16:337-339.

36. Cherukuru R, Menon J, Patel K, Thambidurai R, Subbiah K, Shan- mugam NP, et al. Uncommon presentation of a recurrent diaphragmatic hernia after pediatric liver transplantation. Pediatr Transplant 2020;24:e13790. 